\title{
LE TEMPS CÉRÉMONIEL A LA TÉLÉVISION OU LA NOSTALGIE PROGRAMMÉE
}

\author{
Mário Mesquita ${ }^{2}$
}

Pas d'époque où on n'ait pas regretté à cor et à cri la disparition de nos "vraies" fêtes d'antan: la nostalgie est programmée d'avance. Nous entrons dans nos fêtes à reculons. Entrées royales, sacres, funérailles, carrousels, miroitent dans le rétroviseur républicain.

Régis Debray ${ }^{3}$

La médiatisation des cérémonies publiques est un domaine d'étude récent. A la fin des années 70, Elihu Katz et Daniel Dayan ont entamé des projets de recherche sur un objet qui, au début, était

1 Cet article est le résultat de recherches effectuées dans le cadre du Département de communication de l'Université catholique de Louvain. L'auteur remercie l'Université Nouvelle de Lisbonne pour les facilités accordées et la Junta Nacional de Investigação Científica e Tecnológica (JNICT) -institution officielle portugaise de recherche scientifique et technologique- pour l'aide apportée, dans le cadre du programme «Science» (1991-92), qui lui a permis d'entamer cette recherche.

2 Professeur au Département de communication de l'Université Nouvelle de Lisbonne et à la Licence en journalisme de la Faculté de lettres de l'Université de Coimbra.

3 R. DEBRAY, L'État séducteur, Paris, Gallimard, 1993, p. 114. 
construit autour des notions de "diplomatie télévisée" et de "téléévénement"1, puis s'est élargi au concept, plus audacieux, d"événements médiatiques" ("media events").

Il s'agit, selon leur définition, d'événements exceptionnels qui ont la force suffisante pour "interrompre la séquence habituelle de la programmation des télévisions", faisant appel au direct télévisé. Dayan et Katz ont essayé d'identifier un "genre télévisuel", mais bien sûr les cérémonies publiques peuvent prendre d'autres formes de mise en média, même quand il s'agit de la télévision, notamment le traitement dans le cadre du journal télévisé.

L'expression "télécérémonie" apparaît, dans les articles de Dayan et Katz, comme l'équivalent en langue française (nous n'oserons pas dire "la traduction") de "media events" 2 . Il semble juste de remarquer que, si la notion de "télécérémonie" recouvre bien des événements tels qu'un mariage royal ou l'investiture d'un président, le fait qu'elle puisse s'appliquer, par exemple, aux débats télévisés ou aux compétitions sportives paraît moins évident.

D'ailleurs, Daniel Dayan rend compte, dans un texte récent, de l'écart entre les deux concepts évoqués, en écrivant que les cérémonies télévisées "forment(...) un ensemble hétérogène", puisque "certaines d'entre elles appartiennent de longue date à un corpus cérémoniel et parviennent à la télévision déjà dotées d'une dramaturgie consacrée", tandis que "d'autres, plus innovantes, se constituent pour solenniser des situations ou des actes qui n'ont a priori rien de cérémoniel"3.

"Événements médiatiques" ("media events") est évidemment un concept plus large et plus flou que celui de "télécérémonies". Dayan et Katz caractérisent les "événements médiatiques" par trois C: compétition, célébration et conquête. Par "compétition" ils entendent le "combat réglé", qu'il s'agisse des duels télévisés électoraux ou des compétitions sportives. La "célébration" concerne les

1 Cf. D. DayAn et E. Katz (avec P. Motyl), "Diplomatie télévisée: Sadate à Jérusalem", Les Cahiers de la Communication, vol. 2, n² 2, Paris, 1982, p. 83-104. Les études sur la visite de Sadate à Jérusalem (1977) signalent le début du travail du groupe de recherche sur les "media events", selon l'information publiée dans le livre qui représente la synthèse de quinze années de recherches: D. DAYAN et E. KATZ, Media Events. The Live Broadcasting of History, Cambridge-Londres, Harvard University Press, 1992, p. 295.

2 Cf. D. DAYAN et E. KATZ, "Rituels publics à usage privé: métamorphose télévisée d'un mariage royal", in Annales ESC, janv.-févr. 1983 (38 année, $\left.\mathrm{n}^{\circ} 1\right)$, p. 3-20.

3 D. DAYAN, "Cérémonies télévisées", in L. SFEz, Dictionnaire critique de la communication, Paris, P.U.F.,1993, p. 1009. 
"couronnements", y compris les mariages royaux et les funérailles des dirigeants politiques ou religieux. La "conquête" comprend tout le champ des exploits scientifiques et technologiques ${ }^{1}$. L'expression "télécérémonie" semble plus adéquate à la sous-catégorie "célébration", puisqu'elle concerne des manifestations traditionnellement classifiées dans le domaine du rituel politique, civique ou religieux ${ }^{2}$.

Les "événements médiatiques" correspondent à un ensemble de caractéristiques communes sur le plan sémantique, syntactique et pragmatique. D'un point de vue sémantique, les cérémonies se présentent comme la célébration d'un consensus. D'une perspective syntactique, il s'agit d'interruptions de la programmation habituelle de la télévision. Observées sous l'angle pragmatique, les télécérémonies permettent que "l'espace public se réalise à domicile" -elles créent des "devoirs" et des "obligations", dont le devoir de les regarder. Il s'agit donc d'un "performatif cérémoniel"3. Le monde est censé s'adapter à la parole. L'état de choses auquel les télécérémonies font référence est virtuellement réalisé par l'énonciation même.

L'analyse des télécérémonies, selon Katz et Dayan, permet de décrire l'événement comme un processus. L'entité organisatrice (l'État ou tout autre institution), le public direct, les médias (la télévision) et le public atteint par la télévision sont les articulations essentielles de l'événement. L'intervention des différents acteurs se distribue, en général, selon la "chaîne de prises en charges" suivante: a) le rituel originel et ses organisateurs; b) l'État (ou l'institution organisatrice); c) le public direct; d) l'adoption par la TV; e) le public téléspectateur; f) la presse écrite nationale; g) les chaînes de TV étrangères; h) le public téléspectateur étranger; i) la presse écrite étrangère.

La construction d'une télécérémonie suppose l'accord préalable de certains centres de pouvoir -dont l'État, le public (direct et indirect) et les médias- qui célèbrent entre eux un "contrat de communication". Ce contrat peut être refusé par l'une des trois parties ou violé quand un des trois protagonistes principaux se trouve éliminé ou remplacé de façon autoritaire.

1 D. Dayan et E. Katz, Media Events..., op. cit., (surtout le chapitre ${ }^{\circ} 2$, intitulé «Scripting Media Events: Contest, Conquest, Coronation», p. 25-53).

2 Notre article est centré sur les télécérémonies au sens restreint, c'est-à-dire les célébrations.

3 D. DAYAN et E. KATZ, "La télévision et la rhétorique des grandes cérémonies", in Film et histoire, M. FERro (éd.), Paris, École des Hautes Études, 1984, p. 84. 
Le rôle du public dans une télécérémonie soulève un certain nombre de questions: est-il vraiment un partenaire ou est-ce qu'il se limite à ratifier une cérémonie créée par les autres organisateurs, à savoir l'État et les médias? Dans cette perspective, le concept de contrat de réception est particulièrement important: "Recevoir ce type d'événement est un acte social. C'est recevoir l'événement chez soi. C'est aussi, au sens mondain du terme, recevoir. L'événement est ainsi l'occasion d'innombrables invitations à venir regarder la télévision, provoque une "diaspora" de réunions. Il fait l'objet, entre participants à ces réunions, d'un contrat spectatoriel ou, en termes plus vastes, d'un «contrat de réception»" 1 . Mais, évidemment, le public téléspectateur peut aussi se refuser à "participer". Parmi les différentes formes que ce refus peut avoir, il faut citer le cas du "zapping", surtout dans les pays où on a accès à une considérable diversité de chaînes de télévision.

Du point de vue de l'énonciation filmique, les cérémonies s'éloignent d'autres types de récits d'information, dont le principal "genre journalistique" à la télévision: le journal télévisé. La stratégie de l'énonciation cérémonielle, au contraire de celle du journal télévisé, consiste à en effacer les traces d'énonciation pour mettre l'accent sur ce qui arrive aux personnages ou ce que racontent des narrateurs explicites et "actorialisés".

La vieille distinction de Benveniste entre "discours" et "histoire", si discutée au niveau de la sémiotique, devient ici opératoire, dans la mesure où elle oppose, comme le dit Francesco Casetti, d'un côté (discours) "un dire qui manifeste ses propres paramètres de référence (c'est-à-dire le qui, le où et le quand de l'acte qui l'initialise)" et, de l'autre (histoire), "un dire qui fonctionne comme s'il était en dehors de toute place déterminée"2. Casetti souligne que "ces distinctions opposent donc des propositions qui révèlent leur propre volonté d'énonciation et des propositions qui n'ont ni le besoin ni la volonté de le faire"3.

L'analyse du dispositif d'énonciation des télécérémonies, décrit par l'équipe de chercheurs des "media events", permet de vérifier qu'en général les marques d'adresse aux destinataires sont absentes.

1 Ibidem, p. 93.

2 Fr. CASETTI, D'un regard l'autre. Le film et son spectateur, Lyon, Presses Universitaires de Lyon, 1990, p. 46.

3 Ibidem. Casetti emploie le pluriel puisqu'il se réfère aussi à la distinction (analogue) entre «commentaire» et «récit» proposée par Bettetini. 
C'est le régime iconique que Jean-Pierre Meunier décrit comme celui de "l'énonciation off": "les différents personnages, les énonciateurs" des médias -notamment les commentateurs, les interviewers, les interviewés-sont souvent invisibles à l'écran ou sur l'image"'2.

D'habitude le présentateur des télécérémonies n'est pas visible, sa présence étant limitée à une "voix-over", tandis que, par exemple, dans le journal télévisé le présentateur joue le rôle central, regarde le téléspectateur les yeux dans les yeux (axe y-y) et montre (ou simule) une distanciation vis-à-vis des nouvelles qu'il transmet ${ }^{3}$. C'est le régime de "l'énonciation on", selon la terminologie de Meunier, où "(les énonciateurs) sont visibles, c'est-à-dire que d'une certaine manière ils s'opposent aux spectateurs auxquels ils s'adressent directement, ou indirectement comme c'est le cas dans l'interview"4.

Ce régime d'énonciation sublimée convient bien à des dispositifs dont la signification correspond, en général, à un acte performatif du pouvoir. Hors des feux de la rampe, déguisée en maître de cérémonies, cachée sous la voix, pleine d'accents confidentiels, du locuteur ou journaliste, la télévision peut accomplir son rôle avec plus d'efficacité.

Il faut pourtant se garder de généraliser. Une analyse approfondie de plusieurs cérémonies télévisées révélera peut-être une alternance ou une coexistence de différents types d'énonciation. Parfois, deux régimes cohabitent à l'intérieur d'une même "genre", tel que l'a montré Pierre Sorlin à propos du cinéma de fiction dans son analyse sur les films de Griffith, ou "histoire" et "discours" "tendent à coexister sans qu' intervienne jamais un choix clair entre les deux"5.

1 L'expression "énonciateur" employée pour désigner le présentateur du JT ou l'interviewer à la télévision pose, à notre avis, certains problèmes, puisqu'elle est, d'une certaine façon, l'équivalent d'un "narrateur visualisé" au niveau du récit de fiction. On se demande donc s'il ne serait pas préférable de réserver le mot "énonciateur" pour signifier les "instances abstraites et implicites" (Casetti) responsables par l'énonciation dans sa globalité, essayant de trouver une autre désignation -celle de médiateur, par exemple- pour les "instances explicites", c'està-dire celles qui représentent, au niveau du médiatique l'équivalent de ce qu'on appelle "narrateur délégué" (Gaudreault et Jost) dans le récit.

2 J.-P. MEUNIER et D. PERAYA, Introduction aux théories de la communication, Bruxelles, De Boeck Université, 1993, p. 249.

3 E. VÉron, "Il est là, je le vois, il me parle", in Communications, n 38,1983 , p. 98120.

4 J.-P. MEUNIER et D. PERAYA, Introduction..., op. cit., p. 259.

5 P. Sorlin, "L'énonciation de l'histoire", in J. MotTET (éd.), Griffith, Paris, Ramsay, 1989, p. 299. 
Il y a parfois des différences entre les stratégies énonciatives des différentes télévisions. La tendance générale va dans le sens de cacher les marques d'énonciation, mais il arrive, par exemple, que les chaînes décident de rendre visible leur propre appareil technique de production.

C'est le cas de la transmission en direct des funérailles (environ sept heures) de l'ex-premier ministre portugais Sá Carneiro (1980) où, plusieurs fois au long du trajet, les journalistes montrent les caméras, les grues et, en général, le dispositif de production, essayant d'exhiber les moyens techniques de la R.T.P. (Radio Télévision Portugaise) ${ }^{1}$. Mais, pour tout le reste, y compris le schéma de présentation basée dans la "voix over", les télécérémonies partagent le même schéma d'énonciation, proche de la "transparence" du cinéma de fiction, dont le principal signe est l'interdit de regard à la caméra, afin d'éviter la rupture de l'univers fictionnalisant qu'on est en train de créer.

\section{Une origine lointaine}

On pourrait tracer le parcours historique de la réception iconique des cérémonies à partir de la peinture. Quel rapport entre la représentation du cérémoniel dans la Reddition de Breda, de Velázquez, et sa représentation filmique? Il y a certainement au moins l'idée d'imposition d'un ordre rituel qui se manifeste et se prolonge dans le langage iconique, bien avant l'émergence du cinéma et de la télévision.

On n'aura pas, en tout cas, l'ambition de refaire ici l'archéologie du "genre" télécérémonie. D'ailleurs, à l'intérieur de la communication sociale, il y a aussi tout une histoire du cérémoniel, soit au niveau du récit de presse, soit au niveau du radiophonique. Mais on s'intéressera surtout aux cérémonies filmées, puis télévisées.

Katz et Dayan remarquent, inspirés par Benjamin, que les cérémonies télévisées correspondent à la transition d'un "dispositif théâtral" vers un "dispositif cinématographique": "l'on assiste au déclin

1 On peut s'interroger sur la raison d'être de cette stratégie. Les réalisateurs et les journalistes, que nous avons interviewés, le justifient surtout à cause de la gestion des "temps morts", typiques de la situation cérémonielle. On peut suggérer que cette option relève surtout d'une sorte d'exhibitionnisme technologique très répandu à la télévision. "Narcisisme de la machine" c'est l'expression employée, à ce propos, par Chr. METZ, op.cit., p. 92. 
progressif d'une publicité que l'on croyait aller de soi, d'une «publicité» caractérisée par la rencontre effective des acteurs cérémoniels et de leur public en des lieux géographiquement définissables: parlements, esplanades, églises, congrès. Cette «publicité» de type «théâtral» est remplacée par un nouveau mode de publicité, «fondée» cette fois-ci sur la séparation potentielle des acteurs et de leurs publics et de l'indétermination qui en résulte quant à une géographie de l'événement, sur l'impact des rhétoriques narratives substitué aux vertus du contact. Ce nouveau mode de «publicité» n'est plus thêâtral. Il s'inspire du cinéma"1.

En effet, la transition des cérémonies publiques du "dispositif théâtral" -dans les rues, les places ou les palais- vers le "dispositif cinématographique" appartenait déjà à l'histoire du cinéma avant d'entrer dans l'histoire de la télévision.

Les cérémonies publiques sont liées au cinéma presque dès ses premiers jours: François Doublier, opérateur au service des frères Lumière, a filmé en Russie, "Le couronnement du czar Nicolas II" (1896). Du point de vue des Lumière, créateurs du cinéma-documentaire, il s'agissait d'une reproduction d'images d'un rituel d'État, mais pour Georges Méliès, père fondateur du cinéma de l'illusion, du trucage et de la magie, il faudra ouvrir un autre chapitre: celui des "cérémonies reconstruites".

Aucun document ne signale mieux la qualité de double artefact de la représentation filmique des cérémonies que celle du couronnement du Roi Edouard VII d'Angleterre, réalisée par Méliès (1902). Appuyé par le chef du protocole de la cour britannique, il a reconstruit la cathédrale de Westminster, dans les studios de Montreuil, en France, afin de filmer la cérémonie par anticipation.

Les autorités britanniques ont refusé l'accès des caméras à Westminster, mais elles ont donné leur accord à la représentation fictionnelle, où les rôles du roi et de la reine furent joués par des amateurs. Le couronnement anticipé par Méliès fut exhibé à Londres, avec succès, avant la cérémonie authentique elle-même. Le Roi a vu le film et, selon la légende, a félicité Méliès pour avoir montré les parties de la cérémonie qui n'ont pas existé dans le réel².

1 D. Dayan et E. KaTZ, "Rituels publics...", op. cit., p. 3.

2 M. Huret, Ciné actualités. Histoire de la presse filmée (1895-1980), Paris, Henri Veyrier, 1984, p. 24-25. 
Le public savait "parfaitement qu'on lui offrait une sorte d'imagerie analogue à celle d'un illustrateur et non la reproduction d'une réalité vraie", explique Jean Mitry ${ }^{1}$. Mais l'interprétation de Mitry sera bientôt révisée: la reproduction deviendra la vraie cérémonie. La reproduction (la réplique) sera plus importante que l'original.

Le célébre "Triomphe de la Volonté", de Lenni Riefensthal (1934), montre bien la primauté de la construction filmique sur le spectacle lui-même ${ }^{2}$. La logique des télécérémonies était déjà inscrite dans le film de Riefensthal. Ce n'était pas un documentaire sur le Congrès nazi de Nuremberg, puisque le congrès avait été préparé afin de produire le film ${ }^{3}$.

Ces films de "propagande politique" traduisaient la rhétorique des régimes totalitaires des années 40 . On peut les considérer comme des précurseurs des télécérémonies contemporaines, en tant que solutions de compromis entre le récit journalistique et la mise en scène qui dépasse le simple enregistrement des faits ${ }^{4}$.

Les télécérémonies ne s'intègrent pas dans le concept de reportage journalistique, et ne se situent pas non plus au même niveau du "récit factuel", car, comme le souligne Daniel Dayan, «l'événement transmis ne se présente pas comme le constat d'un état de fait, mais comme un acte discursif (dont la rhétorique peut changer) ou une construction culturelle (dont les règles peuvent évoluer)"5.

Le couronnement de la Reine Elisabeth II (1953) a marqué le début de la télévision en tant que moyen de diffusion de masse en Angleterre et en Europe. Plusieurs familles ont acheté leur premier poste de télévision afin d'assister en direct à la cérémonie ${ }^{6}$. Les caméras ont été autorisées pour la première fois à entrer à Westminster.

1 J. Mitry, Histoire du cinéma, t. 1, (1895-1915), Paris, Éditions Universitaires, 1968, p. 206.

2 S. KraCAUER, De Caligari à Hitler, Une histoire du cinéma allemand, (19191933), Paris, Flammarion, 1987, p. 330.

3 Ibidem, p. 344.

4 Ibidem.

5 D. DAYAN, "Présentation du Pape en voyageur. Télévision, expérience rituelle, dramaturgie politique", in Terrain, $\mathrm{n}^{\circ} 15$, oct. 1990, p. 15.

6 "Mes parents ont acheté un appareil de télévision, comme beaucoup d'autres personnes dans le pays, afin de voir le couronnement de 1953...", raconte l'ancien directeur-général de la BBC, Al. MILNE in DG: The memoirs of a British Broadcaster, Londres, Hodder \& Stoughton, 1988, p. 5. 
Certains commentateurs ont parlé d'un double couronnement: le couronnement de la Reine et celui de la télévision ${ }^{1}$.

Dans un film de fiction récent, on a reconstitué l'adhésion des téléspectateurs britanniques à la cérémonie royale de $1953^{2}$, sous la forme d'un "comité de réception" constitué dans le "living room" de la famille d'un libraire de Londres. Le comportement de cette petite audience devant leur poste de télévision montrait la façon -on dirait presque religieuse- dont l'ambiance cérémonielle était transférée vers le domicile privé. Le sommet de ce climat d'émotion arrive quand tous les présents se lèvent de leurs chaises afin d'entendre et de chanter, debout, le "God Save the Queen".

Cette reconstitution appartient au modèle d'un téléspectateurcitoyen, qui se sent dans le devoir d'adhérer aux rites civiques, à l'aide du petit écran, tel un croyant religieux participe dans un acte de culte. La télévision s'associe au "sense of occasion", à la fête civique ou religieuse, ou à la nostalgie pour la tradition des grandes commémorations religieuses ou civiques ${ }^{3}$.

A l'image du couronnement de 1953, certaines cérémonies publiques, ayant des potentialités dramatiques suffisantes pour être promues au niveau d'“événements médiatiques", ont franchi les frontières du pays d'origine et obtenu les plus grandes audiences télévisées de toujours. C'est le cas des transmissions en direct des funérailles de John Kennedy et du mariage du Prince Charles et de Lady Diana, dont l'audience internationale peut être calculée au niveau des 500 millions de téléspectateurs ${ }^{4}$.

Ces moments d'une espèce de ritualité médiatisée par la télévision ne furent pas, bien entendu, l'exclusivité des démocraties occidentales. Les cérémonies publiques télévisées dans les pays de l'ancien "bloc socialiste" ont aussi joué un rôle dans la légitimation de ces régimes au niveau interne et externe. "J'ai adhéré pleinement à

1 A. BRIGGS, The history of broadcasting in the United Kingdom, Oxford, Oxford University Press, vol. IV, 1979, p. 458-459.

2 Il s'agit du film 84 Charing Cross Road, réalisé par David Jones (USA, 1987), dont le scénario a été inspiré par les mémoires de l'écrivain Hélène Hanff.

3 Cf. E. KATZ, "Os acontecimentos mediáticos: o sentido de ocasião", in N. TRAQUINA (org.), Jornalismo: Questões, Teorias e 'Estórias', Lisbonne, Vega, 1993 , p. 53 et passim ("Media events: the sense of occasion", in Studies in Visual Communication, $\mathrm{n}^{\circ} 6,1980$, p. 84-89).

4 Cette information est donnée par D. DAYAN et E. KATZ, in Media Events ..., op. cit., p. 127. Néanmoins, les auteurs soulignent qu'il s'agit d'un nombre approximatif. 
l'image de la Russie de Gagarine. Pour d'autres, ce fut le couronnement de la Reine Elizabeth; chez nous, on a acheté la télé pour suivre la visite en France de Khrouchtchev", a déclaré M. Robert Hue, secrétaire national du $\mathrm{PCF}^{1}$. Ce témoignage exemplifie bien la projection internationale de ce type d'événement cérémoniel.

\section{Un genre ambigu}

Peut-on classer les cérémonies télévisées dans la catégorie des documentaires? On a déjà entamé la réponse, à propos de l'oscillation entre Lumière et Méliès, qu'un certain personnage de Jean-Luc Godard, dans La Chinoise, a traité ironiquement, en disant que Méliès est le fondateur du documentaire et Lumière le père du cinéma de fiction.

Compte tenu de l'ironie, il faut y revenir de façon plus approfondie. Le terme documentaire désigne, au sens large, "toute œuvre cinématographique, ne relevant pas de la fiction, qui s'attache à décrire ou à restituer le réel"2.

Au niveau des procédés de langage, il y a une grande homologie entre les films de fiction et de non-fiction. L'emboîtement des discours, l'existence de différentes instances discursives, la cohabitation de plusieurs matières d'expression sont des tendances communes aux deux grandes branches du cinéma.

Les genres non narratifs, souligne Christian Metz, "puisent dans le même répertoire de figures énonciatives, mais en les traitant autrement: que l'on songe, par exemple à la voix-in avec regard-caméra dans les informations télévisées, et à la façon dont elle alterne avec la voix-off dans les brefs reportages enchâssés"3.

On doit même nuancer la distinction entre les films narratifs et non narratifs, car il existe -Metz lui-même le reconnaît- "des films partiellement narratifs, et même à tous les degrés"4.

1 J.-L. SAUX, "Ce bon docteur Hue", in Le Monde, 28 mars 1995, p. 15.

2 Définition empruntée à R. BASSAN, in J.-L. PASSEK (éd.), Dictionnaire du cinéma, Paris, Larousse, 1986, p. 189.

3 Chr. METz, L'énonciation impersonnelle ou le site du film, Paris, Méridiens Klincksieck, 1991, p. 181.

4 M. MARIE et M. Vernet, "Entretien avec Christian Metz", in Iris, no 10 (Christian Metz et la théorie du cinéma), Méridiens Klincksieck, p. 219. 
Le traitement télévisé des cérémonie publiques renvoie au vieux problème du statut du film documentaire par rapport au film de fiction. D'ailleurs, la dichotomie "documentaire-fiction" est loin de correspondre à un statut défini et consensuel, au niveau des théoriciens du cinéma. "Tout film (est) un film de fiction", a écrit Metz" "Tout film de fiction (peut) être considéré, d'un certain point de vue, comme un documentaire"2, affirme Odin. Ces affirmations, apparemment contradictoires, signifient, au fond, que "tout film participe à la fois des deux régimes"3.

C'est dans ce sens que Jean Renoir disait, à propos de son film de fiction La Règle du Jeu, qu'il n'était "qu'un documentaire sur le marquis de La Chesnaye" (personnage du film). Francis Vanoye a commenté, du point de vue de l'analyse filmique, l'affirmation du réalisateur: "Le film comporte les caractéristiques esthétiques et éthiques d'un documentaire: respect du réel qu'on s'efforce d'enregistrer, exploration systématique et exhaustive de la réalité qu'on a choisi de présenter (ici: des lieux -hôtel particulier, château, bois,-, des activités -lever, chasse, fête-, des sentiments, etc.), neutralité relative du regard"4.

Mais si le film de fiction se présente, parfois, comme un documentaire, il faut noter que le film dit non narratif -par exemple, le film scientifique, le film pédagogique ou le reportage- "tombent sous cette loi qui veut que par ses matières de l'expression (image mouvante, son) tout film irréalise ce qu'il représente et le transforme en spectacle"s.

Soit le film scientifique, soit le film documentaire se réfèrent à une réalité extra-filmique, mais "ils nous présentent des aspects inconnus de la réalité qui relèvent ainsi plus de l'imaginaire que du réel"6. André Bazin a analysé ce "paradoxe du documentaire" à propos d'un film sur une expédition scientifique: "Ce requin baleine entrevu dans les reflets de l'eau nous intéresse-t-il par la rareté de l'animal et du spectacle -mais on le distingue à peine!- ou plutôt parce que l'image a été prise dans le même temps où un caprice du

\footnotetext{
1 A. Gaudreault et Fr. Jost, Le récit cinématographique, Paris, Nathan, 1990, p. 31 .

2 R. ODIN, "Film documentaire, lecture documentarisante", in Cinéma et réalités, Saint-Étienne, (J.-C. LYANT et R. ODIN éd.), Université, Cierec, 1984, p. 263.

3 A. Gaudreault et Fr. Jost, op.cit., p. 31.

4 Fr. VANOYE, La règle du jeu. Jean Renoir, Paris, Nathan, 1989, p. 73.

5 J. Aumont et alii, Esthétique du film, Paris, Nathan, 1983, p. 71.

6 Ibidem.
} 
monstre pouvait anéantir le navire et envoyer la caméra et l'opérateur par 7000 ou 8000 mètres de fond? La réponse est facile: ce n'est pas tant la photographie du requin que celle du danger"1.

Ce n'est pas le danger qu'on retient dans les télécérémonies. On y filme peut-être la conjuration d'un danger, l'effort pour exorciser un malaise, une angoisse diffuse dans nos sociétés habituées à vivre en crise permanente au niveau politique, social et économique. On enregistre donc la révérence, le respect, la dévotion face aux instances organisatrices des cérémonies qui se manifestent dans ces moments de ritualité. On soumet la caméra aux impératifs du cérémoniel.

Le mélange de fiction et documentaire dans les télécérémonies commence dans le référent qu'on est supposé rapporter avec fidélité. Méliès est donc invoqué par Dayan et Katz à propos de la célèbre visite de Mitterrand au Panthéon: "Le président pénètre dans le mausolée, tenant à la main ce qui est devenu l'emblème du Parti Socialiste pendant la campagne présidentielle: une rose rouge. A l'intérieur du mausolée, il réussit à fleurir, à tour de rôle, trois tombes. Le vainqueur socialiste se doit d'entrer au Panthéon une rose à la main. L'héritier de plusieurs des grandes traditions françaises se doit de rendre hommage à plusieurs sépultures. Concilier ces multiples exigences symboliques, implique pourtant que l'on aménage le tournage en direct, le réalisme de l'événement. Il faut que les quelques minutes consacrées par le président au recueillement soient également consacrées au remplacement des roses. Dotée d'une incontestable grandeur, cette incursion solennelle dans l'au-delà, cette conversation silencieuse avec les mentors défunts, ne réalise pleinement sa vocation rituelle que sur l'écran. Le Panthéon est devenu studio. Par le miracle des roses, un hommage supplémentaire plane sur la cérémonie: un autre grand mort se trouve célébré: Georges Méliès"2.

La distinction entre la fiction et le documentaire, les "genres" narratifs et non narratifs est elle-même problématique. "Un documentaire se définit comme présentant des êtres et des choses existant positivement dans la réalité afilmique"3. On peut alors demander, avec pertinence, si, dans le cas de notre objet d'étude, l'événement originel -la cérémonie elle-même- appartient à la "réalité afilmique", c'est-àdire "la réalité qui existe dans le monde usuel, indépendamment de

1 A. BAZIN, Qu'est-ce que le cinéma?, Paris, Cerf, 1985, p. 32.

2 D. DAYAN et E. KATZ, "Rituels publics...", op. cit., p. 4.

3 Ét. Souriau, apud A. Gaudreault et Fr. Jost, op.cit., p. 34. 
tout rapport avec l'art filmique"1, ou si, au contraire, étant construite en fonction de la médiatisation télévisuelle, elle s'insère déjà, au départ, dans un monde presque fictionnel.

Daniel Dayan considère aussi que les cérémonies télévisées en direct sont presque des membres de la même famille du roman réaliste du XIX" siècle: "Les événements dont retentit l'histoire se déroulent par la télévision ou grâce à sa présence. Mis ensemble ils forment des continuités: littéralement des séries télévisées. Sadate sort de son avion; parle à la Knesset; signe les accords de Camp David; assiste à un défilé militaire; s'écroule. Kennedy surpasse Nixon en éloquence et en photogénie; visite Dallas; périt. Le Prince de Galles tombe amoureux d'une divorcée, l'épouse, abdique; Elisabeth est couronnée; Margaret se marie; Mountbatten est enterré; le prince Charles est déclaré prince héritier... La famille royale britannique vit une existence de feuilleton, l'équivalent en vrai de la saga des Forsythe... L'histoire devenue fiction, se transforme en enchevêtrement d'épisodes à suivre, dont la plupart communiquent entre eux suivant la technique mise au point par les romanciers anglais du XIX ${ }^{\mathrm{e}}$ siècle pour authentifier leurs intrigues"2. Dans cette perspective -selon Dayan- "la télévision reprend à son compte les stratégies de Balzac ou, plus crûment, celles de Tolstoï de Guerre et paix qui n'hésitait pas à mettre en scène Koutouzov ou Napoléon"3.

Les comparaisons osées de Katz et Dayan entre les télécérémonies et le "kino-pravda" ou le roman réaliste, sont discutables et lointaines, mais elles montrent bien qu'il s'agit d'un "réel" construit, d'une sorte de théâtre filmé. Même celles qui procèdent d'une attitude de reportage, au niveau du travail du réalisateur, ou des journalistes, n'échappent pas au sortilège de l'artefact au service de la célébration.

L'information journalistique est basée dans le conflit et dans la critique, tandis que la célébration cérémonielle a pour objectif la création d'un consensus. Certes, le journaliste peut osciller entre l'adhésion et la distanciation critique, mais le dispositif cérémoniel dans lequel il est intégré réduit au minimum la chance d'un “adversarial journalism" dans le cadre d'une cérémonie publique. D'habitude, "le style habituel des journalistes cède la place au lyrisme

Ibidem.

2 D. DAYAN, "La comédie humaine par satellite", in Le Nouvel Observateur, $\mathrm{n}^{\circ}$ spécial, "Littérature et Audiovisuel" (mars-avril 1982), p. 46.

3 Ibidem. 
(...). La prose informative des commentateurs se transforme en une poésie de célébration ${ }^{1}$.

Cela est bien documenté au niveau de l'analyse de certaines cérémonies, comme c'est le cas des funérailles du Roi Baudouin. "Si le public a participé au deuil, les journalistes aussi y ont participé, ce que certains leur ont reproché", écrit Marc Lits². Laissant ouvert le débat éthique des journalistes, Lits écrit ce propos: "On peut (...) se demander s'il est possible de montrer l'émotion sans y céder. Si l'information, lors de ces journées c'était l'émotion du public, était-il possible de la dire, de la faire percevoir, sans utiliser le langage de l'émotion?"'.

Le rituel convoque l'unanimité. L'observateur neutre inscrit dans la déontologie journalistique est, du point de vue de l'organisateur de la télécérémonie, une figure plus qu'indésirable, inconcevable. Le récit cérémoniel est, par définition, une espèce de cercle carré de la déontologie journalistique, le lieu géométrique de l'anti-journalisme, dans la mesure où il réduit radicalement toute hypothèse de neutralité ou de critique.

"A part l'un ou l'autre, tout le monde a eu envie de participer, vu l'ampleur de l'événement", a déclaré, dans le cadre de l'enquête menée par Benoît Grevisse, un journaliste belge à propos des funérailles du Roi $^{4}$. Même si "participer" n'est pas synonyme de "reporter", puiqu'il s'agit aussi de s'engager, de communier d'un état d'esprit collectif...

"Journalists became priests", , soulignent Katz et Dayan, c'est-àdire deviennent des prêtres, des officiants du rite, intégrés dans l'engrenage de la rhétorique cérémonielle, avec une voie très étroite entre l'adhésion et le rejet. Dans plusieurs analyses de télécérémonies, effectuées par l'équipe de Dayan et Katz, sont présentes les références à la situation paradoxale des journalistes: "L'équipe énonciative de la télévision n'est plus, ni-dessus, ni en dehors de l'événement. Elle fait (...) partie d'une histoire racontée dans le style du cinéma de fiction"6.

1 D. DAYAN et E. KATZ, "Cérémonies télévisées", in Médiaspouvoirs, n 12, 1988, p. 27.

2 M. LITS, "Édition spéciale: le roi est mort", in M. LITS (éd.) Le roi est mort...Émotion et médias, Bruxelles, Vie Ouvrière, 1994, p. 24.

3 Ibidem, p. 22.

4 B. GREvisse, “...depuis la mort de Kennedy. Lectures de récits de pratiques journalistiques", in M. LiTs (éd.), op. cit., p. 170.

5 D. Dayan et E. KaTZ, Media Events ..., op. cit., p. 192. On lira, sur ce thème, le sous-chapitre "Effects on Journalists and Broadcasting Organizations", p. 192-195.

6 D. DAYAN et E. KATZ, "Cérémonies télévisées", op. cit., n 12, 1988, p. 30. 


\section{Des ilmages d'archives présentifiées}

Les cérémonies politiques cohabitent dans les écrans de télévision avec des actes de communication politique moderne, voire de marketing politique. Dans le temps télévisé, il s'agit de mondes voisins, qui peuvent même se confondre aux yeux du spectateur distrait, mais qu'une analyse plus fine permet de distinguer: la gestion du temps d'une cérémonie publique obéit à des règles bien distinctes de celles qui réglementent le temps télévisé de l'information (journal télévisé) ou de la publicité.

Est-ce que la dimension rituelle des cérémonies résiste au temps de divertissement et de futilité qui est, en général celui de la TV? Estce que la dimension symbolique des cérémonies peut survivre, côtoyant les spots publicitaires, les feuilletons, les journaux télévisés, dans le continuum de l'écran télévisé? Nous retiendrons deux points de vue opposés qui prennent comme exemple le même type de cérémonie: les voyages du Pape.

D'un côté, c'est Daniel Dayan qui analyse la retransmission télévisée des voyages du Pape comme un "rite de passage". Pour lui, la télévision permet la ritualisation "non plus de la quête du sacré -le voyage du pèlerin- mais sa réception qui prend alors la forme d'un adventus". Le medium télévisuel "fait appel à la plus forte des scansions disponibles: la désorganisation volontaire de la grille des programmes"1.

Du point de vue de Dayan, les voyages du Pape traduisent "une politique esthétique consistant à maintenir l'aura et la distance des cérémonies dont il est l'officiant; à établir que celles-ci sont avant tout destinées aux congrégations massées sur les lieux de célébration, et qu'elles n'atteignent qu'accessoirement le public des téléspectateurs"2 .

De l'autre côté, il y a Serge Daney, selon lequel la télévision n'est pas capable de scander le temps, d'installer un temps autre, celui du rite. Au contraire, elle détruit la valeur symbolique des "pèlerinages" du Pape: "Si est symbolique tout acte qui scande la durée et instaure le temps pour un groupe d'individus qui -de ce fait même- se reconnaît embarqué sur un seul et même esquif, la télévision a, a eu et aura bien du mal à créer de tels actes. Paradoxalement, c'est son omniprésence qui la rend sans pouvoir. Mondiale et permanente, elle ne

1 D. Dayan, «Présentation du Pape..., op. cit., p. 19.

2 Ibidem, p. 15. 
scande plus rien. Zappable et corvéable à merci, elle ignore la catharsis. Dénuée de hors-champ, elle est aussi sans autrui. D'où de redoutables effets pervers. Tel événement qui, hier encore, passait pour «symbolique» (disons le voyage du Pape) devient une pure parodie dans la grande tradition pince-sans-rire des derniers films de Buñuel"1.

La nature de la médiatisation télévisée des cérémonies contemporaines renvoie aux conceptions générales sur la société, le système politique, le sacré et le profane, l'image et les médias. Si l'on partage un certain type de jugement sur la télévision selon lequel elle "dévalorise tout ce qu'elle touche par une mise à plat des différences, des sens et des symboles" naturellement réduites à la dimension d'un élément, parmi d'autres, dans un flot ininterrompu d'images télévisées, où la règle est l'insignifiance et la banalisation.

Dans cette perspective, la médiatisation des cérémonies a pour effet la destruction de ce que Walter Benjamin appelle, dans un essai célèbre, l"“aura" qui se joue dans "le ici et maintenant de l'œuvre d'art, -l'unicité de sa présence au lieu où elle se trouve"3. Dans ce cas, on peut se référer à l'“aura" de la cérémonie.

Au cours d'analyses sur les cérémonies de "disparition" de Giscard d'Estaing et d'“apparition" de François Mitterrand", de la décoration posthume des morts de Beyrouth aux Invalides 5 ou de la messe télévisée ${ }^{6}$, Daney défend que la télévision n'a aucune possibilité de nous restituer un temps rituel.

"La télévision a inventé ses propres rituels (les jeux, les débats) -écrit Daney- mais claudique un peu dès qu'il est question de se greffer sur des rituels pré-existants, historiques, ancrés dans l'histoire de France. Des rituels qui ont déjà été peints ou gravés. La télé, en effet, a plutôt tendance à tout rendre trivial, à zoomer publicitairement sur n'importe quoi, à tout rapetisser (d'où son nom de «petit écran»). Alors, devant une messe, une visite d'un Président aux morts du Panthéon ou une sonnerie aux morts, elle redécouvre que l'instrument

1 S. DANEY, Devant la recrudescence des vols de sacs à main, Lyon, Aléas Éditeur, 1991, p. 169.

2 J.-L. MissiKA et D. Wolton, La folle du logis, Paris, Gallimard, 1983, p. 166.

3 W. BENJAMIN, "L'œuvre d'art à l'ère de sa reproductivité technique", in Essais 2 (1935-1940), Paris, Denoël-Gonthier, 1983, p. 90.

4 S. DANEY," Ciné-Journal", Cahiers du Cinéma, 1986, p. 23-27.

5 Ibidem, p. 185-187.

6 S. DANEY, Le salaire du zappeur, Paris, Ramsay, 1988, p. 222-226. 
qu'elle a en mains n'est pas innocent ou que, comme on dit (en vain) depuis des siècles: les formes sont lourdes de contenu"1.

Par contre, Daniel Dayan et Elihu Katz proposent une autre interprétation des cérémonies télévisées. Leur démarche est presque une apologie des télécérémonies ${ }^{2}$ en tant que fait communicationnel. Selon eux, il s'agit d'événements télévisés qui possèdent "un caractère performatif", puisqu'ils proposent "sous une forme cérémonielle une modification ou un renforcement des valeurs centrales de la société"3. D'après cette conception, les cérémonies télévisées ont un rôle d'intégration sociale et nationale, puisqu'elles se présentent comme la célébration d'un consensus, comme réaffirmation (ou réarticulation) de principaux critères d'identité collective.

Quel que soit le malaise expérimenté par la télévision face à la transcription de ce genre d'événements, c'est un fait que, parmi les points de repère dans l'histoire de la télévision, il y a couronnements et mariages royaux, rites d'investiture des Présidents de la République, visites d'État, "pèlerinages" du Pape, funérailles nationales et d'autres cérémonies médiatisées.

Les télécérémonies se trouvent, en quelque sorte, à l'intersection de trois temporalités de la télévision: le temps de l'information, le temps de la fiction et le temps des archives.

Les cérémonies télévisées sont proches du temps d'information dans la mesure où elles se présentent comme un temps de reportage, ayant recours à la technique du "direct", qui représente, aujourd'hui un élément essentiel de l'information télévisée. D'ailleurs, une des premières utilisations du direct à la télévision fût justement celle des cérémonies publiques. Avant d'arriver au temps de l'information et du reportage, la technique du direct a commencé par la cérémonie, comme si le pouvoir politique et médiatique -à l'âge d'or des télévisions publiques- voulait dompter cette nouvelle technique, tout en essayant de la mettre au service d'une ritualité civique inspirée par la tradition.

Les cérémonies télévisées ont aussi des aspects en commun avec le temps de la fiction, puiqu'elles représentent une sorte de théâtre civique ou religieux. Les démocraties comtemporaines, toujours en

1 S. DANEY, Ciné-Journal, op. cit., p. 186.

2 C'est notamment le cas de l'article de E. Katz (avec D. DAYAN et P. MotYL), "In Defense of Media Events", in Communications in the 21st Century, R.W. HAIGH,

G. GERBNER et R.B. BYRNE (éd.), New York, Wiley, 1981, p. 43-59.

3 D. DAYAN et E. KATZ, "Cérémonies télévisées", op. cit., p. 25. 
quête d'une légitimation suplémentaire, ont besoin, si non d'“inventer une tradition" - pour reprendre le concept de l'historien Hobsbawmau moins de la mettre en scène. L'univers des télécérémonies correspond -selon des concepts inspirés à Dayan et Katz par l'anthropologue Victor Turner- à un "monde subjonctif" (le monde est ce qu'il devrait être), tandis que le monde du journal télévisé correspond à 1"'indicatif" (le monde est ce qu'il est) ${ }^{1}$.

Le temps cérémoniel à la télévision représente aussi une sorte d'archives présentifiées. Des vieilles images, devenues pâles par le décours des années, sont réconfigurées et rajeunies par la couleur de la télévision. Ces images des archives de l'imaginaire historique deviennent le décor où des acteurs contemporains jouent leurs rôles, tout en essayant de chercher dans les cérémonies d'autrefois un supplément de légitimité.

Toutefois, si le temps cérémoniel présente des analogies avec les régimes de l'information, de la fiction et des archives, il se singularise par son objectif de suspendre le temps quotidien, soit au niveau interne de la télévision, soit au niveau de la réception.

Au niveau de la gestion du temps de la production, les télécérémonies introduisent, à la fois, une rupture avec la grille de la programation télévisée et une autre coupure avec la logique de vitesse et de fragmentation de l'information télévisée.

Si les temps de l'information et de la publicité à la télévision sont marqués par l'urgence et par le culte de l'instantané, au contraire, le temps cérémoniel se caractérise par un rythme compassé.

Le journal télévisé "supprime la dimension temporelle de l'événement", puisque les images, "extraites de leur séquence (...), n'obéissent plus au rythme de l'événement, mais deviennent plutôt ses illustrations, ses emblèmes"2. Par contre, les télécérémonies assurent une continuité temporelle, elles "sont traitées comme des récits continus", dans la mesure où "la cohérence syntagmatique de l'émission se calque sur la progression de l'événement lui-même de façon d'autant plus évidente que l'événement est transmis en direct"3.

Une certaine esthétique cérémonielle vient ajouter ses exigences aux contraintes technologiques du direct: "L'événement étant filmé en «direct», doit l'être sans coupes, toute ellipse impliquant alors un trou

1 D. DAYAN et E. KATZ, Media Events..., op. cit., p. 104.

2 D. DAYAN et E. KATZ, "Cérémonies télévisées", op. cit., p. 29.

3 Ibidem. 
-expliquent Dayan et Katz- (...). Le traitement de l'événement inverse la pratique de montage héritée du cinéma -une pratique «substractive»-pour en faire au contraire une pratique «cumulative». L'événement n'est pas allégé de ses éléments insignifiants ou mal venus. Il est doté de prolongements harmoniques"1.

Face au temps fragmentaire de l'information, les télécérémonies instaurent donc la discipline d'un temps continu. Si le journal télévisé correspond à une chronologie de l'immédiat, de l'instant, les cérémonies instaurent une espèce de longue durée télévisée, qui signifie une autre échelle du temps.

Même si les cérémonies civiques et politiques transcrites par la télévision ne correspondent pas à des rites au sens fort du terme, elles constituent un prolongement médiatique de certains "moments de ritualité" 2 civique ou religieuse.

La mise en média des cérémonies télévisées mime la lenteur et la répétition propres du temps rituel. En certaines occasions de dramatisation, les cérémonies télévisions imposent au désordre propre du temps de divertissement télévisé l'ordre inhérent au temps du rituel, essayant d'introduire dans le petit écran -à côté des concours, des jeux et des "infos"- quelques bribes d'un sacré diffus mais unificateur.

Quel sera le destin réservé au cérémoniel civique dans les médias contemporains? Même s'il faut reconnaître que l'idée d'un citoyentéléspectateur paraît en crise, les cérémonies auront peut-être encore un rôle de lien social à l'intérieur de l'espace national, européen ou même international.

Apparemment, la métaphore du rétroviseur ${ }^{3}$ reste opérationnelle. A côté des nouveaux rites créés par la télévision -au niveau du politique (les télédébats) ou du divertissement (les concours, les "reality shows")- les cérémonies héritières d'une mémoire historique et, peut-être, d'une nostalgie d'un sacré lointain reviennent, de temps en temps, afin de bouleverser la grille des chaînes de télévision et le rythme du quotidien des téléspectateurs.

1 D. DAYAN et E. KATZ, "Rituels publics...", op. cit., p. 9.

2 L'expression est empruntée à Marc Augé.

3 Voir le texte de Régis Debray cité en épigraphe de cet article. 